\title{
Flux emergence and interaction with a coronal field: 3D MHD simulations
}

\author{
V. Archontis ${ }^{1}$, F. Moreno-Insertis ${ }^{2}$, K. Galsgaard ${ }^{3}$ \\ and A.W. Hood ${ }^{1}$ \\ ${ }^{1}$ School of Mathematics and Statistics, University of St.Andrews, North Haugh, Fife KY16 \\ 9SS, UK \\ email: vasilis@mcs.st-and.ac.uk, alan@mcs.st-and.ac.uk \\ ${ }^{2}$ Instituto de Astrofisica de Canarias, Via Lactea s/n, 38200 La Laguna, Spain \\ email: fmi@ll.iac.es \\ ${ }^{3}$ Niels Bohr Institute, Juliane Maries Vej 30, 2100 Copenhagen, Denmark \\ email: kg@astro.ku.dk
}

\begin{abstract}
The dynamic process of magnetic flux emergence from the solar interior to the outer atmosphere may well be related with eruptive phenomena and intense events of the Solar activity. However, the physics of the emergence is not still well understood. Thus, we have performed 3D MHD simulations to study the rising motion of a twisted flux tube from the convection zone of the Sun and its interaction with a preexisting coronal magnetic field. The results show that the reconnection process depends criticaly on the initial relative orientation between the two magnetic flux systems into contact. On the other hand, the overal process of emergence depends mostly on the dynamics of the sub-photospheric plasma.
\end{abstract}

Keywords. MHD, magnetic fields, corona

\section{Introduction}

The emergence of active regions from below the solar surface has been observed by the SOHO and TRACE satellites. As the new magnetic flux emerges and expands into the corona, new connections to the preexisting field are seen indicating that effective reconnection occurs between two flux systems with opposite polarity. Intense heating and launching of high-velocity jets follow the reconnection events at the emerging flux region. Furthermore, the coronal magnetic field is destabilized in response to the emergence of magnetic flux, leading to large-scale phenomena such as solar flares and Coronal Mass Ejections (CMEs).

The first numerical simulations, which applied to study the process of flux emergence, were based on two-dimensional models (Yokoyama \& Shibata (1995); Magara (2001) and references therein). A number of three-dimensional models (Fan (2001); Magara \& Longcope (2003); Manchester et al. (2004); Archontis et al. (2004)) have studied the buoyant rise of a flux tube from the convection zone into a nonmagnetized corona. Recently, Fan \& Gibson (2004); Galsgaard et al. (2005); Archontis et al. (2005); Isobe et al. (2005) presented the first three-dimensioanl MHD simulations in which an emerging twisted flux tube interacts with a pre-existing coronal magnetic field.

In the simulations presented in the following we report on the interaction between a buoyant magnetic flux tube and an horizontal ambient field with an orientation that changes between successive experiments. When the two flux systems come into contact a thin arch-like current sheet is formed at the interface. Intricate three dimensional reconnection takes place along the current sheet, so that the corona and the photosphere 

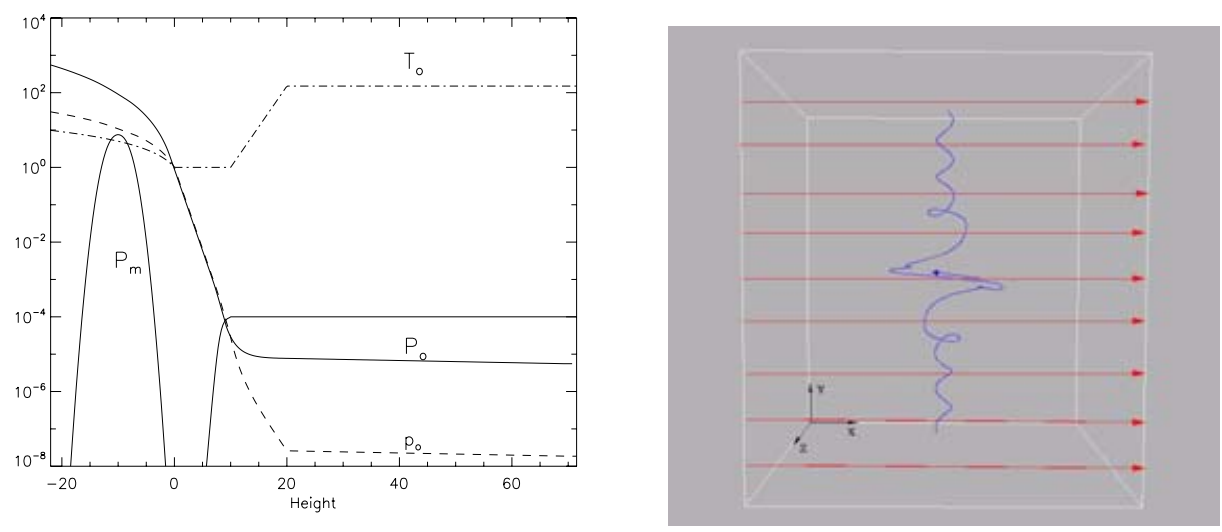

Figure 1. (a) Distribution of temperature (dash-dotted), density (dashed), magnetic pressure (thin solid) and gas pressure (thick solid) along height. Units of length and time are $170 \mathrm{Km}$ and 25 sec respectively.; (b) Top view of an emerging fieldline. The long superimposed arrows show the orientation of the ambient field when $\phi=180$ degrees.

become magnetically connected. A number of striking features are found, such as the formation of plasmoids, fast shocks, intense heating, and hot jets. The present contribution reveals mainly the dependence of the flux emergence process on the relative orientation between the two flux systems.

\section{Initial setup}

The initial setup of the experiments is similar to the previous work of Archontis et al. (2005). The resistive, time-dependent MHD equations (including viscous and ohmic diffusion) are solved in a stretched grid. The computational box consists of an adiabatically stratified layer that represents the convection zone, an isothermal photosphere, a transition region with a steep temperature gradient and an isothermal corona (see Figure $1(a)$ ). A horizontal twisted flux tube is placed $2000 \mathrm{Km}$ below the photosphere. A density deficit is incorporated along the tube (y-direction) and as a result the central part of it becomes underdense and rises faster, developing an $\Omega$-loop shape, at the photosphere.

We use a horizontal and uniform magnetic field for the corona. In fact, the magnetic field strength of the ambient field is $13 \mathrm{G}$ in the corona and transition region (plasma $\beta \approx 0.06$ in the corona) and decreases to zero between the base of the transition region and the middle of the photosphere. The direction of the ambient field is used as a parameter, to study the effect of reconnection on the evolution of the system. If the ambient field is pointing along the positive $x$ - axis then the relative horizontal angle between the two flux systems is $\phi=180$ degrees (see Figure $1(b)$ ), and thus, the relative orientation is favourable for efficient reconnection.

\section{Flux connectivity}

When the two magnetic flux systems come into contact a current concentration is formed at the interface between the rising tube and the coronal field. Full 3D reconnection takes place at the interface and as a result fieldlines are ejected sideways from the current sheet and establish (new) magnetic connections between the corona and the solar interior. To measure the extent to which the two layers become connected, we follow the change of flux connectivity between the tube and the ambient field. The latter is studied by 

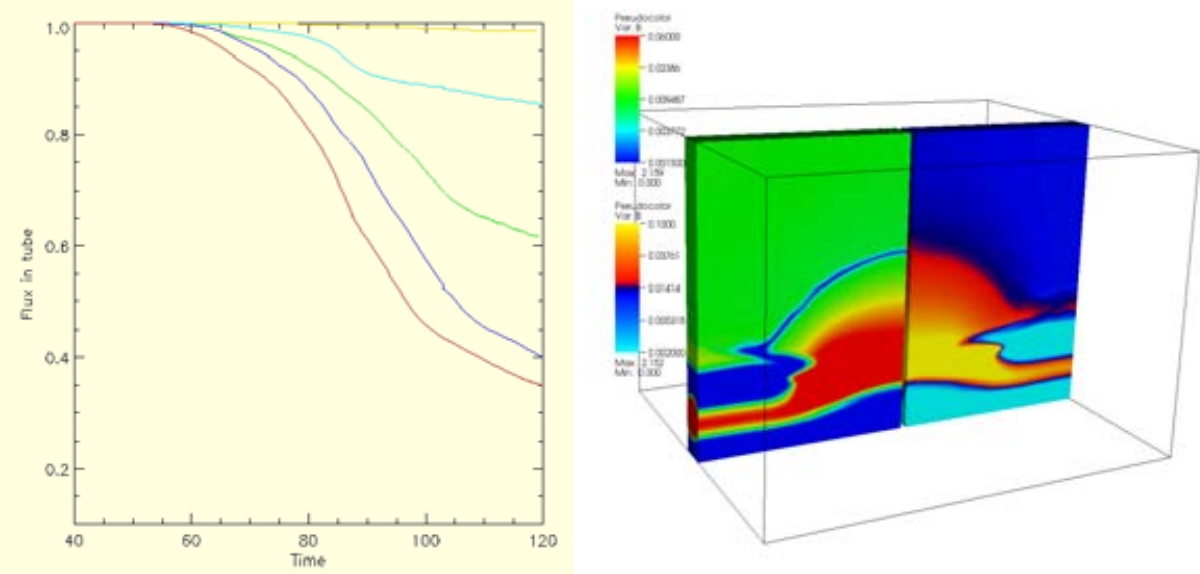

Figure 2. (a) Fraction of magnetic flux that does not change connectivity in time; The five lines correspond to experiments with $\phi=180$ (lower line), 135, 90, 45 and 0 degrees (top line). (b) Colormap of the modulus of the magnetic field vector in two vertical $y z$ midplanes for two experiments $(\phi=180$ (left midplane) and $\phi=45$ (right midplane) ) at $\mathrm{t}=100$.

discriminating between the fieldlines that stay in the tube along the experiment and those that connect to the ambient field (Parnell \& Galsgaard (2004); Archontis et al. $(2005))$.

Figure $2(a)$ shows the amount of flux that remains in the tube as a function of time, normalized to the total initial flux within the tube. The figure shows that there are distinct differences on the flux connectivity as the orientation of the ambient field changes. When the two systems are antiparallel, reconnection is more efficient and the amount of reconnected flux increases up to $65 \%$ by the end of the simulation. On the other hand, when $\phi=0$ (parallel orientation) reconnection is much less efficient and there is almost no change of connectivity. Thus, one may expect that there will also be a difference in the rise of the flux between cases with different ambient field orientations. Figure $2(b)$, however, shows that the apex of the rising tube reaches almost the same height at the same time independently of the relative horizontal angle between the tube and the coronal field. This result indicates that it is possible that emergence depends on the characteristics of the sub-photospheric field and not on the structure or orientation of the corona field.

\section{Reconnection and jets}

As seen in the previous section, the process of reconnection does not play a key-role for the emergence of the tube into the solar atmosphere. However, reconnection is the process through which high-velocity jets (repeatedly observed by the Yohkoh satellite) are formed.

Figure $3(a)$ shows the projection in horizontal plane of high-velocity (Vmax $=150 \mathrm{~km}$ $\mathrm{sec}^{-1}$ ) outflows. These outflows are ejected sideways from the sides of the current sheet, which are marked by the four spots between $-10<x<10$ and $-30<y<20$. The jets are also seen to be regions of high gas pressure and high temperature $\left(810^{5} \mathrm{~K}\right)$. Notice also, that the direction of the magnetic field (white arrows) at the center of the box is 


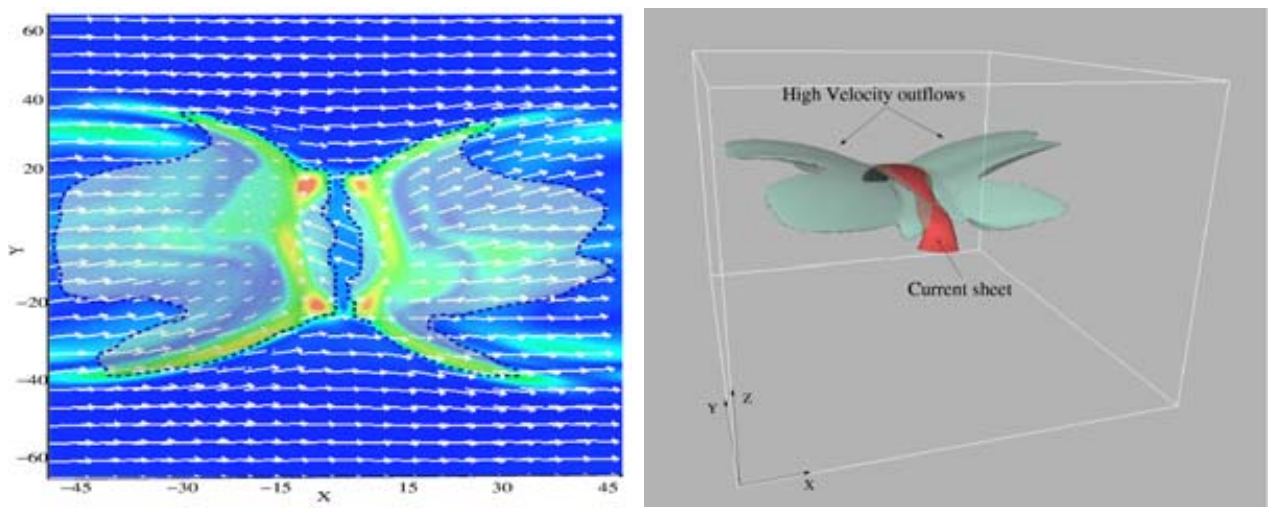

Figure 3. (a) Horizontal cut at $z=2.5 \mathrm{Mm}$ above corona. Shown are the full magnetic field vector (white arrows) and high velocity outflows (transparent isosurfaces) at $t=95.7(b) 3 \mathrm{D}$ visualization of the jets (transparent isosurface) and the current sheet (curved structure between the jets).

not perfectly aligned with the ambient field, although $\phi=180$ at $t=0$. This is because as reconnection proceeds more internal layers of the tube reconnect with the ambient field, but their fieldlines show a shear along height (Archontis et al. (2005)). Figure $3(a)$ shows the full 3D geometry of the jets together with the current sheet. The jets look like curved sheets which are coming from the rims of the current sheet along the $y$-direction and become asymtotically horizontal further away from the interface. This geometry is different to that found in previous 2D models. Finally, we should mention that intense plasma heating due to Joule dissipation is observed in the current sheet when the two flux systems are antiparallel. Values of temperature and current density as high as $10^{7} \mathrm{~K}$ and $200 \mathrm{~A} \mathrm{~cm}^{-2}$ respectively, are found in the current sheet.

\section{Conclusions}

We have provided evidences that although the consequences of the reconnection (flux connectivity, jets, local heating, etc.) change when the relative orientation between the two flux systems varies, the general emergence of the buoyant flux sytem remains unaffected.

\section{References}

Archontis, V., Moreno-Insertis, F., Galsgaard, K., Hood, A.W \& O'Shea, E. 2004, A\&A 426, 1047

Archontis, V., Moreno-Insertis, F., Galsgaard, K., \& Hood, A.W 2005, ApJ 635, 1299

Galsgaard, K., Moreno-Insertis, F., Archontis, V., \& Hood, A.W 2005, ApJ 618, L153

Fan, Y. 2001, ApJ 554, L111

Fan, Y. \& Gibson, S. E. 2004, ApJ 609, 1123

Magara, T. 2001, ApJ 549, 608

Magara, T. \& Longcope, D. W. 2003, ApJ 586, 630

Manchester, W., Gombosi, T., DeZeeuw, D., \& Fan, Y. 2004, ApJ 610, 588

Parnell, C. \& Galsgaard, K. 2004, AAP 428, 596

Isobe, H., Miyagoshi, T., Shibata, K. \& Yokoyama, T. 2005, Nature 434, 478-481

Yokoyama, T. \& Shibata, K. 1995, Nature 375, 42 